\title{
MicroRNA-196a/-196b regulate the progression of hepatocellular carcinoma through modulating the JAK/STAT pathway via targeting SOCS2
}

\author{
Weihua Ren', Shuangting $\mathrm{Wu}^{2}$, Yabin $\mathrm{Wu}^{1}$, Tan $\mathrm{Liu}^{1}$, Xingpeng Zhao ${ }^{1}$ and Yawei $\mathrm{Li}^{3}$
}

\begin{abstract}
microRNAs (miRNAs) play essential roles in progression of hepatocellular carcinoma (HCC). However, the roles of miR196a and miR-196b as well as mechanism in HCC progression remain poorly understood. The expressions of miR-196a, miR-196b and suppressor of cytokine signaling 2 (SOCS2) were measured in HCC tissues and cells by quantitative realtime polymerase chain reaction or immunohistochemistry. HCC progression was investigated by cell proliferation, glycolysis, cycle, clones, apoptosis, and necrosis. The interaction between SOCS2 and miR-196a or miR-196b was explored by luciferase activity and RNA immunoprecipitation analyses. The expressions of proteins in Janus kinase (JAK)/signal transducer and activator of transcription (STAT) pathway were measured by western blot. A xenograft model was established to investigate the roles of miR-196a or miR-196b in vivo. We found that miR-196a and miR196b were highly expressed in HCC tissues and cells. High expression of miR-196a or miR-196b was correlated with tumor size, tumor-node-metastasis stage, lymph node metastasis, albumin-bilirubin grade and poor 5-year survival. Knockdown of miR-196a or miR-196b suppressed cell proliferation, glycolysis, cell cycle process, colony formation but induced apoptosis or necrosis in HCC cells. SOCS2 was targeted by miR-196a and miR-196b and its interference ablated abrogation of miR-196a or miR-196b-mediated inhibitory effect on HCC progression. SOCS2 was negatively associated with activation of the JAK/STAT pathway. Besides, knockdown of miR-196a or miR-196b limited xenograft tumor growth by blocking the JAK/STAT pathway. We concluded that downregulation of miR-196a or miR-196b inhibited HCC progression through regulating the JAK/STAT pathway via targeting SOCS2, providing novel targets for prognosis and therapeutics of HCC.
\end{abstract}

\section{Introduction}

Hepatocellular carcinoma (HCC) is one of the most common malignant tumors and the third leading cause of cancer death with rising incidence worldwide ${ }^{1}$. Despite great advances in diagnosis and treatment of HCC, the recurrence rate remains more than $80 \%$ and the survival

\footnotetext{
Correspondence: Yawei Li (nianxiang0301@126.com)

${ }^{1}$ Medical Diagnostic Centre, Luoyang Central Hospital Affiliated to Zhengzhou University, Luoyang, China

${ }^{2}$ Department of Anesthesiology, Luoyang Central Hospital Affiliated to

Zhengzhou University, Luoyang, China

Full list of author information is available at the end of the article.

Edited by A. Stephanou
}

rate of patients is still unsatisfactory ${ }^{2}$. Hence, it is urgent to explore novel strategies for diagnosis and therapeutics of HCC.

microRNAs (miRNAs) are a class of small noncoding RNAs with $\sim 23$ nucleotides in length, which play essential roles in development and progression of $\mathrm{HCC}^{3}$. miR-196 has been suggested to have an impact on the outcome of development and therapeutics of cancers ${ }^{4}$. Moreover, accruing works have indicated miR-196 as an oncogene to promote progression of various cancers, including glioblastoma, breast cancer, oral cancer, and digestive tract cancers $^{5-8}$. The miR-196 family consists of miR-196a and

\section{(c) The Author(s) 2019}

\footnotetext{
(c) (i) Open Access This article is licensed under a Creative Commons Attribution 4.0 International License, which permits use, sharing, adaptation, distribution and reproduction cc in any medium or format, as long as you give appropriate credit to the original author(s) and the source, provide a link to the Creative Commons license, and indicate if changes were made. The images or other third party material in this article are included in the article's Creative Commons license, unless indicated otherwise in a credit line to the material. If material is not included in the article's Creative Commons license and your intended use is not permitted by statutory regulation or exceeds the permitted use, you will need to obtain permission directly from the copyright holder. To view a copy of this license, visit http://creativecommons.org/licenses/by/4.0/.
} 
miR-196b sharing regulatory efficacy ${ }^{9}$. Previous study has reported that miR-196a is notably upregulated in HCC tissues compared with that in matched nontumor samples $^{10}$. Furthermore, miR-196b has been suggested to be highly expressed in HCC tissues and associated with HCC risk factors ${ }^{11}$. However, little is known about whether and how miR-196a and miR-196b could regulate HCC progression.

The Janus kinase (JAK)/signal transducer and activator of transcription (STAT) pathway has been regarded as one of the main molecular pathways in HCC progres$\operatorname{sion}^{12}$. STAT proteins, including STAT1-6, exhibit promoting or suppressive effect on antiviral response, inflammation, and tumorigenesis in liver, one of which, STAT5, could promote liver damage ${ }^{13}$. Suppressor of cytokine signaling (SOCS) family, such as SOCS1, SOCS2, and SOCS3, has been indicated as key negative regulator of the JAK/STAT signaling pathway in $\mathrm{HCC}^{14-16}$. The available evidence indicated the importance of SOCS2 in various diseases and cancers by regulating biological processes through addressing the JAK/STAT pathway or other signaling ${ }^{17}$. Hence, we hypothesized that miR-196a and miR-196b might regulate progression of HCC by targeting SOCS2. In the present study, we measured the expression of miR-196a and miR-196b and explored their roles as well as underlying mechanism in HCC progression.

\section{Materials and methods}

Patients and tissue samples

A total of 72 patients with HCC who had not received any chemotherapy, radiotherapy, or related treatment before surgery were recruited from Luoyang Central Hospital Affiliated to Zhengzhou University. Written informed consent was obtained from all participants and the work was accepted by the Research Ethics Committee of Luoyang Central Hospital Affiliated to Zhengzhou University. Paired tumor and peri-tumor samples collected from patients were collected for hematoxylin and eosin (H\&E) staining or immediately stored at $-80^{\circ} \mathrm{C}$ until used. The clinicopathological characteristics of patients are shown in Table 1 . The expression data of miR-196a and miR-196b were provided from database of YM500v3 (http://driverdb.tms.cmu.edu.tw/ym500v3/). The 5-year survival rate was investigated in every group. The correlation between clinicopathological characteristics of patients and miR-196a or miR-196b expression is presented in Tables 2 and 3. The liver damage was investigated according to the albumin-bilirubin (ALBI) score $^{18}$.

\section{Cell culture and transfection}

The normal hepatic cell line (LO2) and four HCC cell lines (SMMC-7721, MHCC97-H, Hep3B, and HepG2)
Table 1 The clinic-pathological factors of 72 HCC patients

\begin{tabular}{|c|c|c|}
\hline Clinicopathological feature & Number & $\%$ of patients \\
\hline \multicolumn{3}{|l|}{ Age } \\
\hline$<55$ years & 40 & 55.5 \\
\hline$\geq 55$ years & 32 & 44.5 \\
\hline \multicolumn{3}{|l|}{ Gender } \\
\hline Female & 28 & 38.9 \\
\hline Male & 44 & 61.1 \\
\hline \multicolumn{3}{|l|}{ Tumor size } \\
\hline$>5 \mathrm{~cm}$ & 49 & 68.1 \\
\hline$\leq 5 \mathrm{~cm}$ & 23 & 31.9 \\
\hline \multicolumn{3}{|l|}{$\operatorname{AFP}(\mu \mathrm{g} / \mathrm{L})$} \\
\hline Normal & 34 & 47.2 \\
\hline Abnormal & 38 & 52.3 \\
\hline \multicolumn{3}{|l|}{$\mathrm{HBsAg}$} \\
\hline Present & 52 & 72.2 \\
\hline Absent & 20 & 27.8 \\
\hline \multicolumn{3}{|l|}{ Lymph node metastasis } \\
\hline Yes & 20 & 27.8 \\
\hline No & 52 & 72.2 \\
\hline \multicolumn{3}{|l|}{ TNM stage } \\
\hline$|-| \mid$ & 28 & 38.9 \\
\hline III-IV & 44 & 61.1 \\
\hline \multicolumn{3}{|l|}{ ALBI grade } \\
\hline 1 & 28 & 38.9 \\
\hline 2 & 42 & 58.3 \\
\hline 3 & 2 & 2.8 \\
\hline
\end{tabular}

AFP: alpha-fetoprotein; HBsAg: hepatitis B surface antigen; TNM: tumor-node-metastasis; ALBI: albumin-bilirubin.

were obtained from American Tissue Culture Collection (Manassas, VA, USA). All cells were maintained in Dulbecco's Modified Eagle Medium (Gibco, Carlsbad, CA, USA) containing $10 \%$ fetal bovine serum (Gibco), 100U/ $\mathrm{ml}$ penicillin and $100 \mu \mathrm{g} / \mathrm{ml}$ streptomycin (Invitrogen, Carlsbad, CA, USA) at $37^{\circ} \mathrm{C}$ in a humidified atmosphere with $5 \% \mathrm{CO}_{2}$ during the study.

miR-196a mimic (miR-196a), miR-196b mimic (miR196b), miRNA negative control (miR-NC), miR-196a inhibitor (anti-miR-196a), miR-196b inhibitor (anti-miR196b), miRNA inhibitor negative control (anti-miR-NC), small interfering RNA (siRNA) against SOCS2 (siSOCS2), siRNA negative control (si-NC), pcDNA, or SOCS2 overexpression vector (SOCS2) were synthesized by Genepharma (Shanghai, China). Cell transfection was performed by using Lipofectamine 3000 (Invitrogen) 
Table 2 Correlation between miR-196a expression and clinicopathological characteristics of 72 HCC Patients

\begin{tabular}{|c|c|c|c|}
\hline \multirow[t]{2}{*}{ Clinicopathological feature } & \multicolumn{2}{|c|}{ miR-196a expression } & \multirow[t]{2}{*}{$P$ value } \\
\hline & High $n(\%)$ & Down $n(\%)$ & \\
\hline Age & & & $>0.05$ \\
\hline$<55$ years & $23(57.5)$ & $17(42.5)$ & \\
\hline$\geq 55$ years & $17(53.1)$ & $15(46.9)$ & \\
\hline Gender & & & $>0.05$ \\
\hline Female & $17(60.7)$ & $11(39.3)$ & \\
\hline Male & $23(52.3)$ & $21(47.7)$ & \\
\hline Tumor size & & & $<0.05$ \\
\hline$>5 \mathrm{~cm}$ & $30(61.2)$ & 19 (38.8) & \\
\hline$\leq 5 \mathrm{~cm}$ & $10(43.5)$ & $13(56.5)$ & \\
\hline $\operatorname{AFP}(\mu \mathrm{g} / \mathrm{L})$ & & & $>0.05$ \\
\hline Normal & $19(55.9)$ & $15(44.1)$ & \\
\hline Abnormal & $21(55.3)$ & $17(44.7)$ & \\
\hline $\mathrm{HBsAg}$ & & & $>0.05$ \\
\hline Present & $27(51.9)$ & $25(48.1)$ & \\
\hline Absent & $13(65)$ & $7(35)$ & \\
\hline Lymph node metastasis & & & $<0.05$ \\
\hline Yes & $12(60)$ & $8(40)$ & \\
\hline No & $28(53.8)$ & $24(46.2)$ & \\
\hline TNM stage & & & $<0.05$ \\
\hline$|-| \mid$ & $12(42.9)$ & $16(57.1)$ & \\
\hline III-IV & $28(63.6)$ & $16(36.4)$ & \\
\hline ALBI grade & & & $<0.05$ \\
\hline 1 & $13(46.4)$ & $15(53.6)$ & \\
\hline 2 & $25(59.5)$ & $17(40.5)$ & \\
\hline 3 & $2(100)$ & $0(0)$ & \\
\hline
\end{tabular}

AFP: alpha-fetoprotein; HBsAg: hepatitis B surface antigen; TNM: tumor-node-metastasis; ALBI: albumin-bilirubin.

according to the manufacturer's instructions. Transfection efficacy was evaluated after $24 \mathrm{~h}$ of transfection.

\section{Quantitative real-time polymerase chain reaction (qRT- PCR)}

Total RNA was isolated from tissues or cells by using TRIzol reagent (Invitrogen) following the manufacturer's instructions. The TaqMan microRNA Reverse Transcription Kit (Applied Biosystems, Foster City, CA, USA) or M-MLV Reverse Transcription Kit (Invitrogen) was used for synthesis of complementary DNA (cDNA). The diluted cDNA was amplified using SYBR green (Applied Biosystems) with the following amplification protocol:
Table 3 Correlation between miR-196b expression and clinicopathological characteristics of 72 HCC Patients

\begin{tabular}{|c|c|c|c|}
\hline \multirow[t]{2}{*}{ Clinicopathological feature } & \multicolumn{2}{|c|}{ miR-196b expression } & \multirow[t]{2}{*}{$P$ value } \\
\hline & High $n(\%)$ & Down $n(\%)$ & \\
\hline Age & & & $>0.05$ \\
\hline$<55$ years & $21(52.5)$ & $19(47.5)$ & \\
\hline$\geq 55$ years & $18(56.2)$ & $14(43.8)$ & \\
\hline Gender & & & $>0.05$ \\
\hline Female & $16(57.1)$ & 12(42.9) & \\
\hline Male & $23(52.3)$ & $21(47.7)$ & \\
\hline Tumor size & & & $<0.05$ \\
\hline$>5 \mathrm{~cm}$ & $33(67.3)$ & $16(32.7)$ & \\
\hline$\leq 5 \mathrm{~cm}$ & $6(26.1)$ & $17(73.9)$ & \\
\hline AFP $(\mu \mathrm{g} / \mathrm{L})$ & & & $>0.05$ \\
\hline Normal & $17(50)$ & $17(50)$ & \\
\hline Abnormal & $22(57.9)$ & $16(42.1)$ & \\
\hline $\mathrm{HBsAg}$ & & & $>0.05$ \\
\hline Present & $29(55.8)$ & $23(44.2)$ & \\
\hline Absent & $10(50)$ & $10(50)$ & \\
\hline Lymph node metastasis & & & $<0.05$ \\
\hline Yes & $13(65)$ & $7(35)$ & \\
\hline No & $26(50)$ & $26(50)$ & \\
\hline TNM stage & & & $<0.05$ \\
\hline$|-| \mid$ & $10(35.7)$ & $18(44.3)$ & \\
\hline III-IV & $29(65.9)$ & $15(34.1)$ & \\
\hline ALBI grade & & & $<0.05$ \\
\hline 1 & $12(42.9)$ & $16(57.1)$ & \\
\hline 2 & $25(59.5)$ & $17(40.5)$ & \\
\hline 3 & $2(100)$ & $0(0)$ & \\
\hline
\end{tabular}

AFP: alpha-fetoprotein; HBsAg: hepatitis B surface antigen; TNM: tumor-node-metastasis; ALBI: albumin-bilirubin.

$95^{\circ} \mathrm{C}$ for $10 \mathrm{~min}, 40$ cycles of $95^{\circ} \mathrm{C}$ for $15 \mathrm{~s}$, and $60^{\circ} \mathrm{C}$ for $1 \mathrm{~min}$. The expressions of miR-196a, miR-196b, and SOCS2 were normalized by U6 small RNA or $\beta$-actin with $2^{-\Delta \Delta \mathrm{Ct}}$ method $^{19}$. All primers were obtained from Sangon (Shanghai, China): miR-196a (Forward, 5'- ACCTGCG TAGGTAGTTTCATGT-3' Reverse, 5' -CGTCA GAAGGAATGATGCACAG-3'), miR-196b (Forward, 5'-TAG GTACCACTTTATC CCGTTCACCA-3' Reverse, 5'-AT CTCGAGGCAGGGAGAGAGGAATAA-3'), U6 (Forwar d, 5'-CTCGCTTCGGCAGCACATATACT-3' Reverse, 5'-CGCTTCACG AATTTGCGTGT-3'), SOCS2 (Forward, 5'-GGAACGGCACTGTTCACCTTTA-3' Reverse, $5^{\prime}$-AGCCTACAGAGATGCTGCAGAGA-3'), $\quad \beta$-actin 
(Forward, 5'-CAGC CTTCCTTCTTGGGTAT-3' Rev erse, 5'-TGGCATAGAGGTCTTTACGG-3').

\section{Cell proliferation}

Cell proliferation was investigated by the 3-(4,5-dimethyl-2-thiazolyl)-2,5-diphenyl-2-H-tetrazolium bromide (MTT) assay. SMMC-7721 or HepG2 cells were seeded into 96-well plates at a density of 5000 cells per well. Each group was prepared in triplicate. Cells were interacted with MTT solution (Thermo Fisher, Wilmington, DE, USA) for $4 \mathrm{~h}$ at $0,24,48$, or $72 \mathrm{~h}$. Following the removal of the supernatant, $100 \mu \mathrm{l}$ dimethylsulfoxide (Thermo Fisher) was added to dissolve formazan. The absorbance at $570 \mathrm{~nm}$ was detected using a microplate reader (BioRad, Hercules, CA, USA).

\section{Measurement of glucose consumption and lactate production}

SMMC-7721 and HepG2 cells were seeded in 96-well plates overnight. After $72 \mathrm{~h}$, cells were washed three times in phosphate buffer saline (Gibco), and then collected for analyses of glucose consumption and lactate production via using the Glucose Uptake Colorimetric Assay Kit or Lactate Assay Kit (Sigma, St. Louis, MO, USA) according to the manufacturer's instructions, respectively. The concentrations of glucose consumption and lactate production were measured by using a microplate reader and normalized by the total protein concentration determined via the bicinchoninic acid (BCA) assay kit (Thermo Fisher).

\section{Flow cytometry}

Flow cytometry was conducted to measure cell apoptosis or necrosis. After $72 \mathrm{~h}$, transfected cells were washed with PBS. Cells were resuspended in binding buffer and double stained with the Annexin V-fluorescein isothiocyanate (FITC)/propidium iodide (PI) apoptosis detection kit (Sigma) according to the manufacturer's instructions. The apoptotic or necrotic cells were analyzed through a flow cytometer (Becton Dickinson, Franklin Lakes, NJ, USA) with CellQuest software.

\section{Western blot}

Cells or tissues were lysed in RIPA lysis buffer (Thermo Fisher) containing $1 \%$ protease inhibitor (Sigma). After centrifuged at $16,000 \times g$ for $20 \mathrm{~min}$ at $4{ }^{\circ} \mathrm{C}$, total proteins were quantified by the BCA protein assay kit and then denatured at $98^{\circ} \mathrm{C}$ for $10 \mathrm{~min}$. Proteins were separated by SDS-PAGE gel electrophoresis and transferred to polyvinylidene difluoride membranes (Millipore, Billerica, MA, USA), followed by blocked with $5 \%$ nonfat milk in Tris-buffer saline containing 0.1\% Tween 20 (TBST) for $1 \mathrm{~h}$ at room temperature. Subsequently, membranes were incubated with primary antibodies (1:1000 dilution, Cell
Signaling Technology, Danvers, MA, USA) against B-cell lymphoma-2 (Bcl-2, \#4223), Bcl-2 associated X proteins (Bax, \#5023), SOCS2 (\#2779), STAT5 (\#25656), phosphorylation of STAT5 (p-STAT5, \#4322), JAK2 (\#3230), p-JAK2 (\#3771), or $\beta$-actin (\#4970) overnight at $4{ }^{\circ} \mathrm{C}$. $\beta$-actin was regarded as loading control in this study. After washed with TBST for three times, membranes were incubated with horseradish peroxidase-conjugated secondary antibodies (\#7074, 1:10000 dilution, Cell Signaling Technology) for $2 \mathrm{~h}$ at room temperature. The protein signals were visualized via using enhanced chemiluminescence chromogenic substrate (Thermo Fisher) and analyzed with Image Lab software (Bio-Rad).

\section{Luciferase activity assay}

The putative binding sites of 3' untranslated regions (3'UTR) sequences of SOCS2 and miR-196a or miR-196b were predicted by using TargetScan Release 7.2 (http:// www.targetscan.org/vert_72/). pGL3 vectors (Promega, Madison, WI, USA) were used to synthesize wide-type (SOCS2-WT) or mutant-type (SOCS2-MUT) luciferase reporter vectors containing wild-type or mutant binding sites, respectively. Luciferase reporter vectors and miR196a, miR-196b or miR-NC were cotransfected in HepG2 cells using Lipofectamine 3000 according to the manufacturer's protocols. After the transfection for $48 \mathrm{~h}$, luciferase activity was analyzed by using the luciferase assay kit (Promega) and normalized to Renilla luciferase activity.

\section{RNA immunoprecipitation (RIP)}

The RIP assay was conducted in HepG2 cells by using the RNA-binding protein immunoprecipitation kit (Millipore) according to the manufacturer's protocols. HepG2 cells transfected with miR-196a, miR-196b, or miR-NC were lysed in RIP buffer with anti-Ago2- or IgG-bound magnetic beads. The mRNA level of SOCS2 enriched on beads was measured by qRT-PCR after treatment of TRIzol reagent.

\section{Immunohistochemistry}

Tumor and peri-tumor tissues were fixed with $4 \%$ polyoxymethylene (Sigma), embedded in paraffin and sectioned to $4 \mu \mathrm{m}$ thick. Following blocking endogenous peroxidase using 3\% $\mathrm{H}_{2} \mathrm{O}_{2}$ (Sigma), sections were incubated with primary antibodies against SOCS2 (ab74533, 1:100 dilution, Abcam, Cambridge, UK) for $2 \mathrm{~h}$ and incubated with antirabbit IgG for $30 \mathrm{~min}$, followed by stained with diaminobenzidine and hematoxylin (Sigma). The positive cells were detected under a light microscopy (Olympus).

\section{Murine xenograft model}

$\mathrm{BALB} / \mathrm{c}$ nude mice (male, four-week-old) were obtained from Vital River Laboratory Animal Technology (Beijing, 
China) and housed in specific pathogen-free conditions with a $12 \mathrm{~h}$ light/dark cycle and free access to water and food. Every experiment was made to minimize animals ( $n$ $=7$ per group) under the approval of the Animal Research Committee of Luoyang Central Hospital Affiliated to Zhengzhou University. HepG2 cells were transfected with the lentiviral vectors with anti-miR-196a (Lenti-anti-miR196a), anti-miR-196b (Lenti-anti-miR-196b), anti-miRNC (Lenti-anti-miR-NC), or negative control (Lenti-NC) constructed by GeneCopoeia (Rockville, MD, USA). Stably transfected cells $\left(5 \times 10^{6}\right)$ were subcutaneously injected into the nude mice. Tumor volumes were measured every week for 5 weeks with the formula: volume $\left(\mathrm{mm}^{3}\right)$ $=$ width $^{2} \times$ length $/ 2$. After 5 weeks following the inoculation, the mice were killed and tumor samples were collected and weighted. The collected tumor tissues were used for further molecular studies. Cell apoptosis and proliferation in tumor tissues were measured by terminal deoxynucleotidyl transferase dUTP nick-end labeling (TUNEL) staining with in situ cell death detection kit (Roche, Mannheim, Germany) or Ki67 immunohistochemistry with anti-Ki67 antibody (ab16667, 1:1000 dilution, Abcam) following the manufacturer's instructions as previous study ${ }^{20}$.

\section{Statistical analysis}

The data were presented as the mean \pm standard deviation (S.D.) from three independent experiments. The survival curve was generated via Kaplan-Meier method. The statistical differences between groups were investigated by Student's $t$ test or one-way analysis of variance (ANOVA) using SPSS 18.0 software (SPSS, Inc., Chicago, IL, USA). $P<0.05$ was regarded as statistically significant.

\section{Results}

miR-196a and miR-196b expressions are enhanced and SOCS2 level is decreased in HCC tissues and cells

The morphology investigation of HCC and peri-tumor tissues using H\&E staining displayed that stained HCC tissues showed typical HCC morphology with many formed balloon-like lesions and the peri-tumor tissues exhibited normal liver cell morphology (Fig. S1). Bioinformatics analysis suggested that miR-196a and miR-196b were highly expressed in $\mathrm{HCC}$ tissues compared with that in normal tissues by YM500v3 (Fig. S2A and S2B). To explore the roles of miR-196a and miR-196b in HCC progression, their expression levels were measured in HCC tissues. Compared with peri-tumor group, HCC tissues showed higher expression levels of miR-196a and miR-196b (Fig. 1a, b). Similarly, their levels were also increased in HCC cells compared with those in control group (Fig. 1c, d). Furthermore, patients were classified as high expression and low expression group according to the mean value of miR-196a or miR-196b expression level, named as high miR-196a expression $(n=40)$ and low miR-196a expression $(n=32)$ or high miR-196b expression $(n=39)$ and low miR-196b expression $(n=33)$ group, respectively. Results showed that high expression of miR-196a or miR-196b was associated with tumor size,

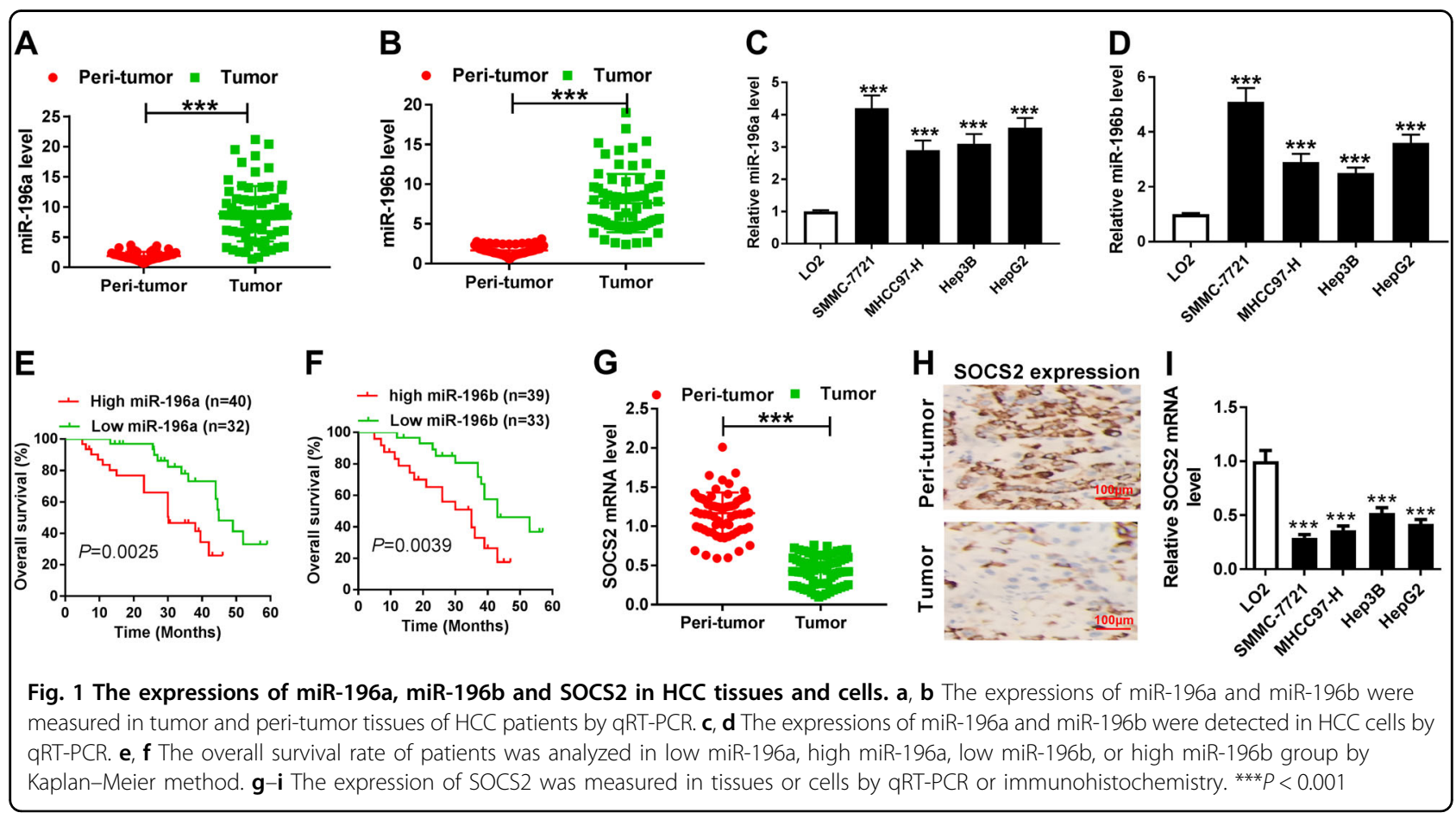



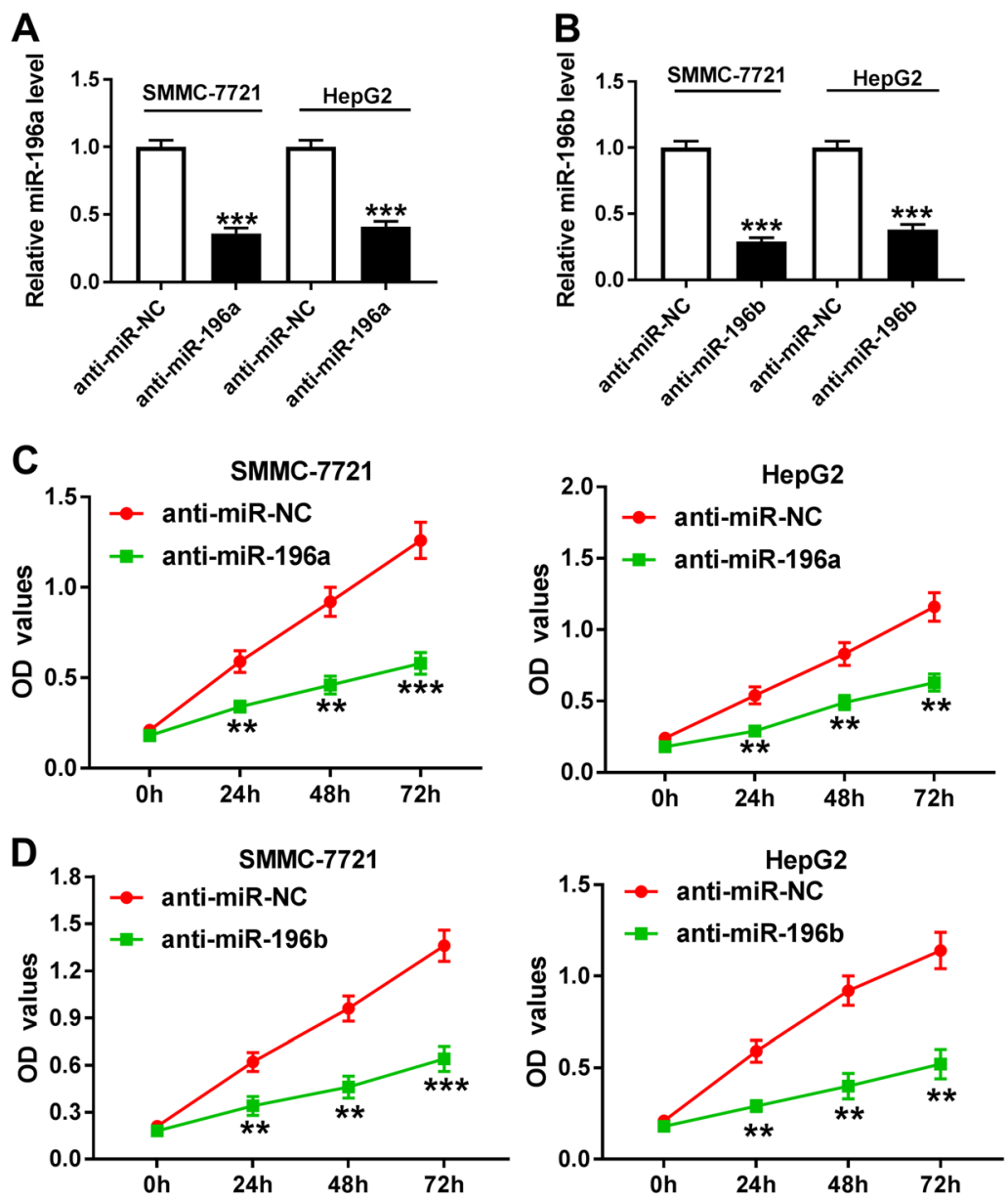

E
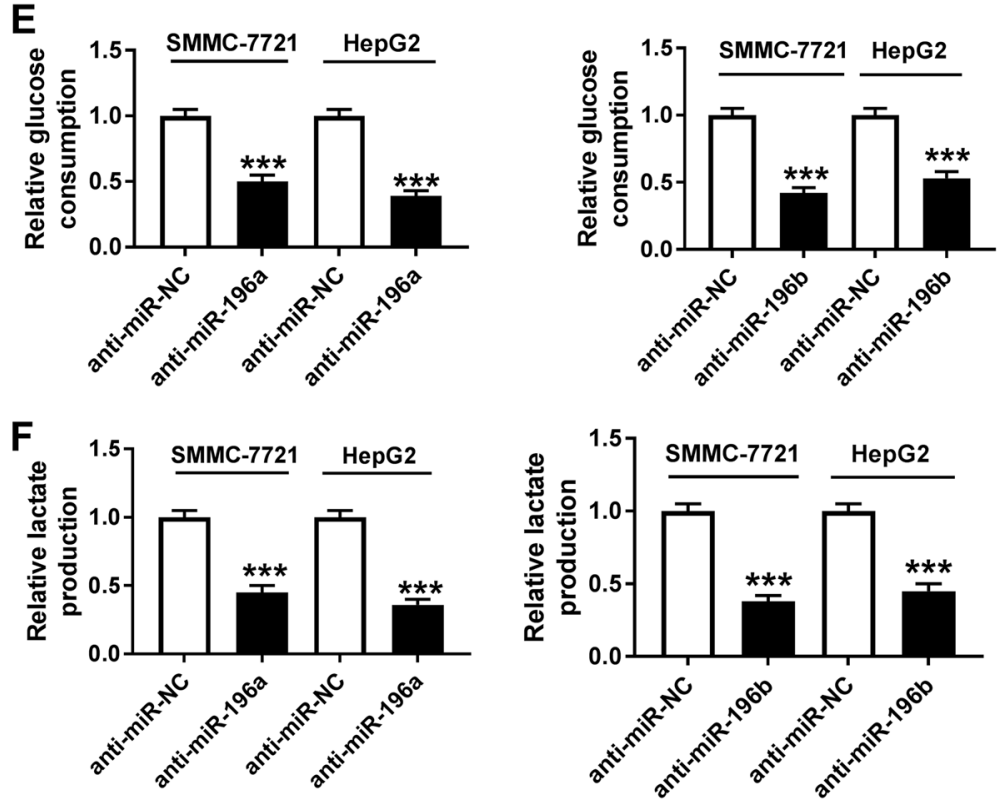

Fig. 2 The effect of miR-196a or miR-196b knockdown on cell proliferation and glycolysis in HCC cells. SMMC-7721 and HepG2 cells were transfected with anti-miR-196a, anti-miR-196b, or their corresponding anti-miR-NC. Their expression levels (a, b), cell proliferation (c, d), glucose consumption and lactate production (e, $\mathbf{f}$ ) were measured in the two cells by qRT-PCR, the MTT assay, or the special commercial kit. ${ }^{* *} P<0.01$, ${ }^{* * *} P<$ 0.001 


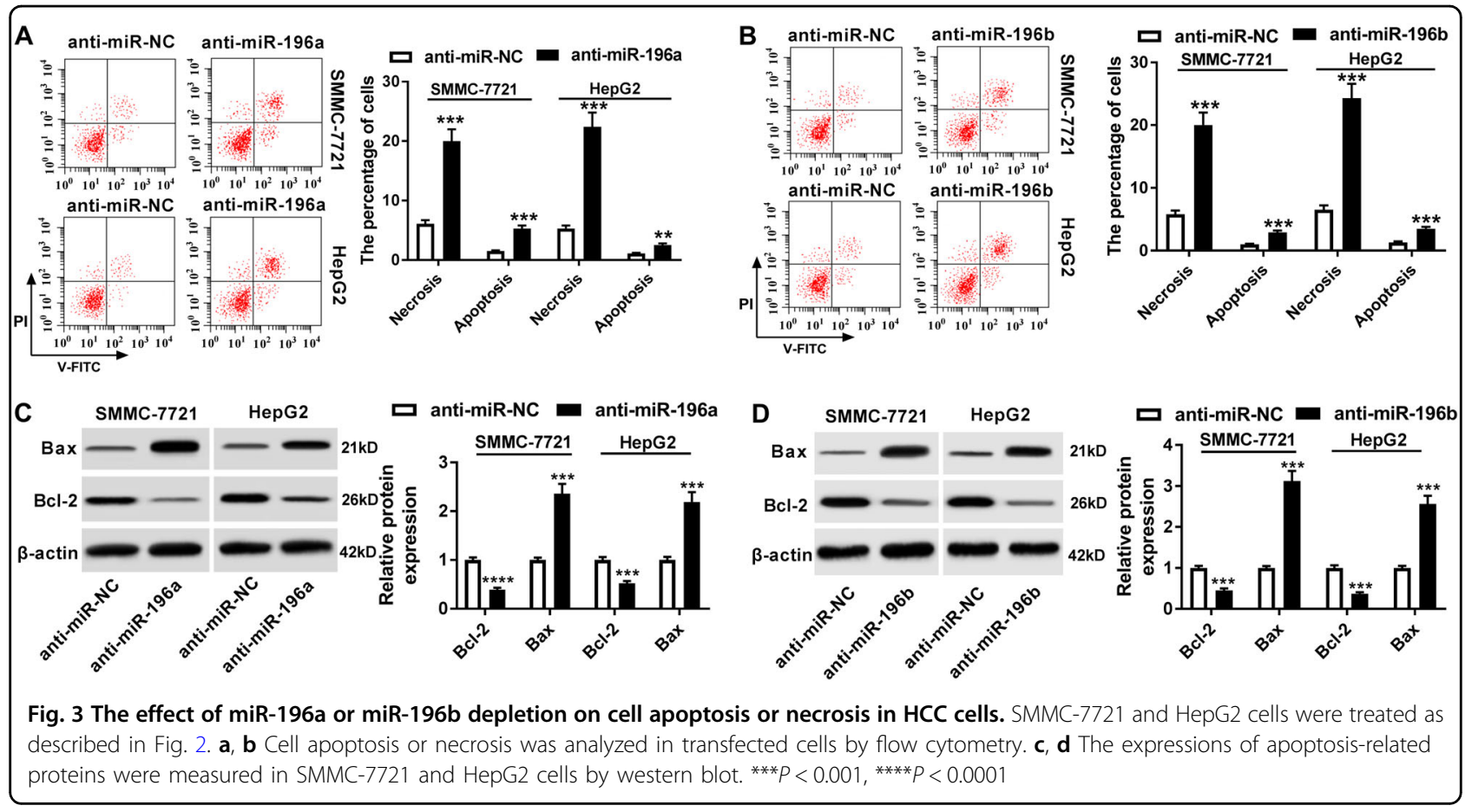

tumor-node-metastasis stage, lymph node metastasis, and ALBI grade (Tables 2, 3 and Fig. S2C-F). In addition, high expression of miR-196a or miR-196b displayed lower overall survival rate $(P=0.0025$ or 0.0039 , respectively) (Fig. 1e, f). Besides, the expression of SOCS2 mRNA and protein were significantly decreased in HCC compared with that in corresponding control (Fig. 1g-i).

\section{Knockdown of miR-196a or miR-196b inhibits progression of HCC cells}

To investigate the potential roles of miR-196a and miR196b in HCC, SMMC-7721 and HepG2 cells with higher abundances of miR-196a and miR-196b were transfected with anti-miR-196a, anti-miR-196b, or anti-miR-NC. The analysis of transfection efficacy showed that the abundances of miR-196a and miR-196b were effectively reduced in the two cells after transfection of anti-miR196a or anti-miR-196b (Fig. 2a, b). Cell function analysis showed that knockdown of miR-196a or miR-196b suppressed HCC progression by inhibiting cell proliferation, glucose consumption and lactate production (Fig. 2c-f), arresting cell at G0/G1 stage (Fig. S3A and S3B) and promoting apoptosis or necrosis through regulating $\mathrm{Bcl}-2$ and Bax (Fig. 3a-d). In addition, by infecting lentiviral vectors with anti-miR-196a (LV-anti-miR-196a), antimiR-196b (LV-anti-miR-196b), or anti-miR-NC (LVanti-miR-NC), the number of clones was obviously decreased in SMMC-7721 and HepG2 cells (Fig. S3C and S3D).

\section{miR-196a and miR-196b regulate HCC progression by} targeting SOCS2

To explore the potential mechanism underlies miR196a or miR-196b participating in progression of HCC, the promising targets were wanted in HCC cells. Bioinformatics analysis provided the potential targets by TargetScan Release 7.2 (Table 4). Therein, SOCS2 with the potential binding sites is an important target involved in pathogenesis of HCC (Fig. 4a, b). To validate this prediction, luciferase activity and RIP analyses were conducted into HepG2 cells. Results showed that overexpression of miR-196a or miR-196b significantly reduced luciferase activity in HepG2 cells transfected with SOCS2-WT, whereas the efficacy was lost in term of SOCS2-MUT (Fig. 4c, d). Moreover, the enrichment of SOCS2 abundance was notably elevated in miR-196a or miR-196b-transfected group compared with that in miRNC group after Ago2 RIP, while IgG failed to show efficacy of enrichment (Fig. 4e, f). Meanwhile, SOCS2 displayed higher ability of miR-196a or miR-196b enrichment than SOCS1 (Fig. S4A and S4B). Moreover, western blot data showed that accumulation of miR-196a or miR-196b limited SOCS2 protein level, while deficiency of miR-196a or miR-196b played an opposite effect (Fig. 4g, h).

To investigate whether SOCS2 was required for miR-196a or miR-196b-mediated progression of HCC, SMMC-7721 and HepG2 cells were cotransfected with anti-miR-196a or anti-miR-196b and si-SOCS2, or si-NC. 
Table 4 The part of predict targets of miR-196a/b from TargetScan Release 7.2

\begin{tabular}{llll}
\hline miR & Target gene & Representative transcript & Gene name \\
\hline miR-196a & SOCS2 & ENST00000548537.1 & Suppressor of cytokine signaling 2 \\
& HMGA2 & ENST00000403681.2 & High mobility group AT-hook 2 \\
& ING5 & ENST00000313552.6 & Inhibitor of growth family, member 5 \\
& HOXC8 & ENST00000040584.4 & Homeobox C8 \\
& FAM127A & ENST00000257013.7 & Family with sequence similarity 127, member A \\
& GALC & ENST00000261304.2 & Galactosylceramidase \\
& ABCB9 & ATP-binding cassette, sub-family B (MDR/TAP), member 9 \\
MAP4K3 & ENST00000280560.8 & Mitogen-activated protein kinase kinase kinase kinase 3 \\
SOCS2 & ENST00000263881.3 & Suppressor of cytokine signaling 2 \\
ABCB9 & ENST00000548537.1 & ATP-binding cassette, sub-family B (MDR/TAP), member 9 \\
MAP4K3 & ENST00000280560.8 & Mitogen-activated protein kinase kinase kinase kinase 3 \\
& TMEM143 & ENST00000263881.3 & Transmembrane protein 143 \\
IQCJ-SCHIP1 & ENST00000293261.3 & IQCJ-SCHIP1 readthrough \\
TBPL1 & ENST00000337808.6 & TBP-like 1 \\
SSR1 & ENST00000237264.4 & Signal sequence receptor, alpha \\
SNAP91 & ENST00000244763.4 & Synaptosomal-associated protein, 91 kDa \\
\hline
\end{tabular}

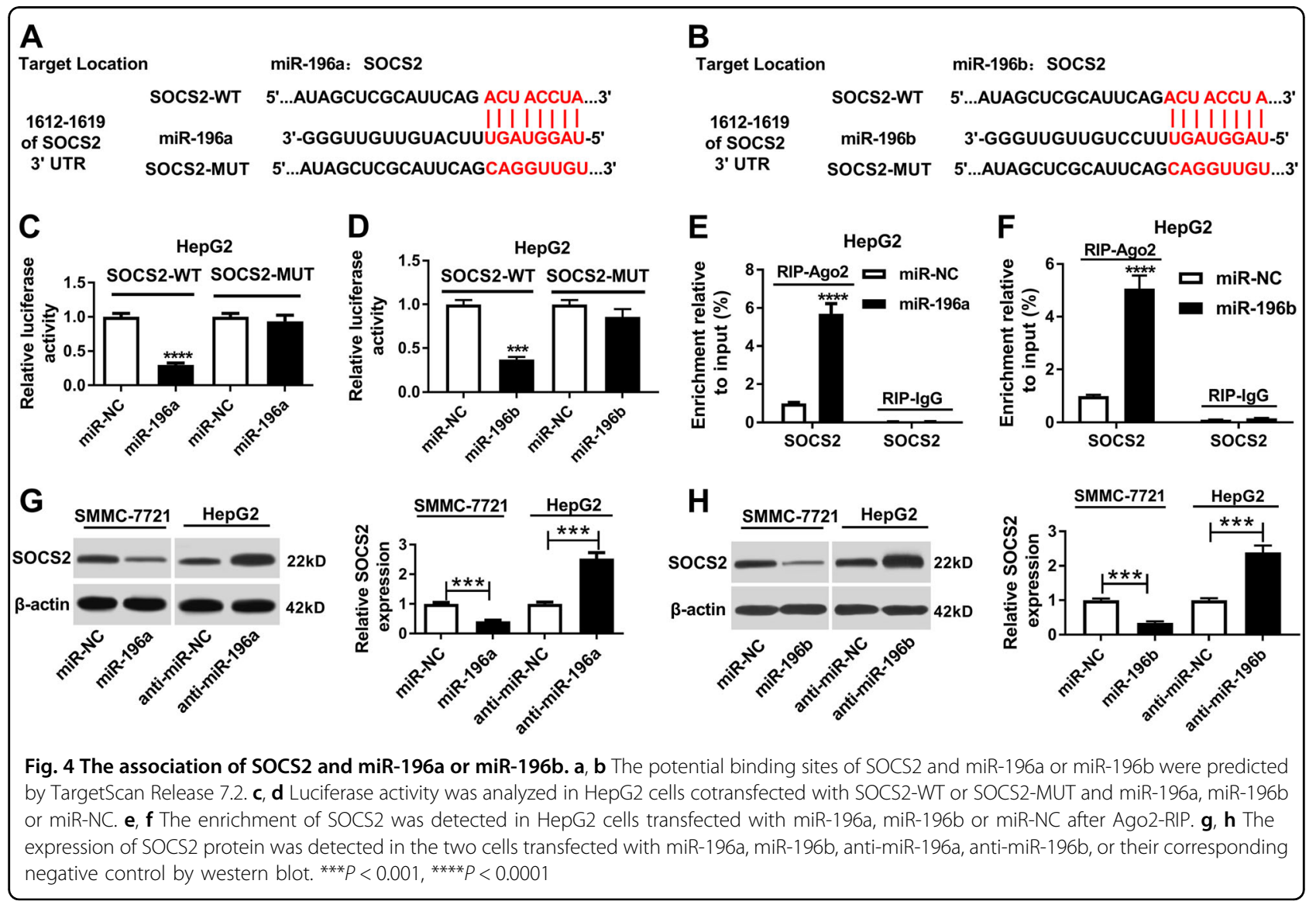




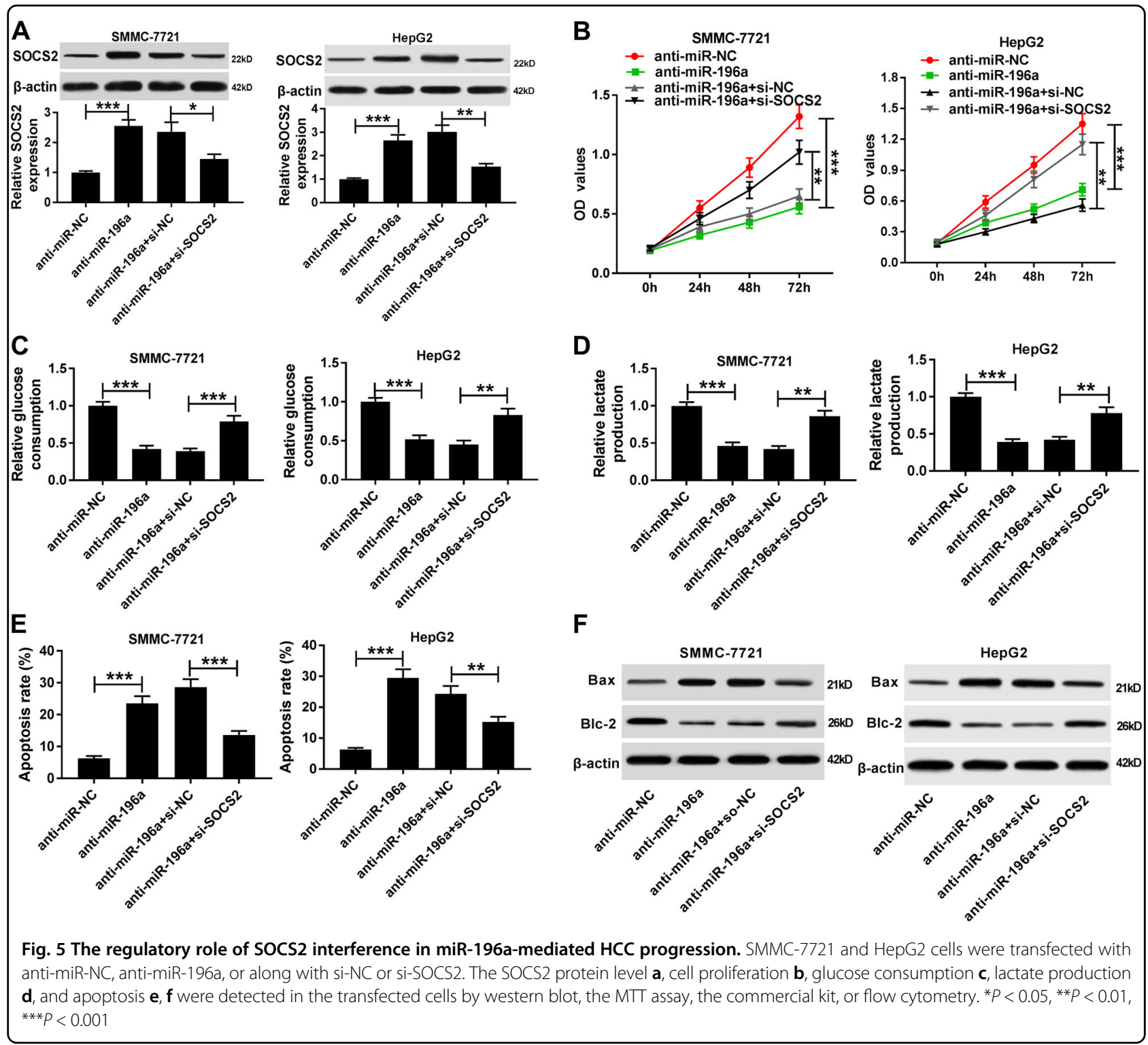

Transfection efficacy was validated in Figs. 5a and 6a. Moreover, recuse experiments displayed that knockdown of SOCS2 reversed downregulation of miR-196a or miR196b-mediated inhibition of cell proliferation, glycolysis, cell cycle progress, colony formation, and apoptosis (Figs. 5b-f, 6b-f, Fig. S5A-D).

\section{Depletion of miR-196a or miR-196b inhibits HCC progression by regulating the JAK/STAT pathway}

To explore the potential signaling pathway participating in $\mathrm{HCC}$ progression in this study, the expressions of $\mathrm{p}$ STAT5, STAT5, p-JAK2, and JAK2 proteins were measured in SMMC-7721 and HepG2 cells transfected with SOCS2, pcDNA, si-SOCS2, or si-NC. As a result, the abundance of SOCS2 protein was effectively elevated by transfection of SOCS2 expression vector and reduced by introduction of si-SOCS2 compared with their corresponding control, respectively (Fig. 7a, b). Moreover, overexpression of SOCS2 inhibited phosphorylation of JAK2 and STAT5, whereas its knockdown promoted the expressions of p-JAK2 and p-STAT5 in SMMC-7721 and HepG2 cells (Fig. 7a, b).

To further investigate the roles of miR-196a and miR196b in HCC, the murine xenograft model was established by subcutaneously injecting with HepG2 cells infected with Lenti-NC, Lenti-anti-miR-NC, Lenti-antimiR-196a, or Lenti-anti-miR-196b. Results displayed that knockdown of miR-196a or miR-196b decreased tumor volume and weight compared with treatment of Lenti-anti-miR-NC (Fig. 8a, b). Then the expression of miR-196a or miR-196b was measured in tumor tissues. Impaired expression of miR-196a or miR-196b was 


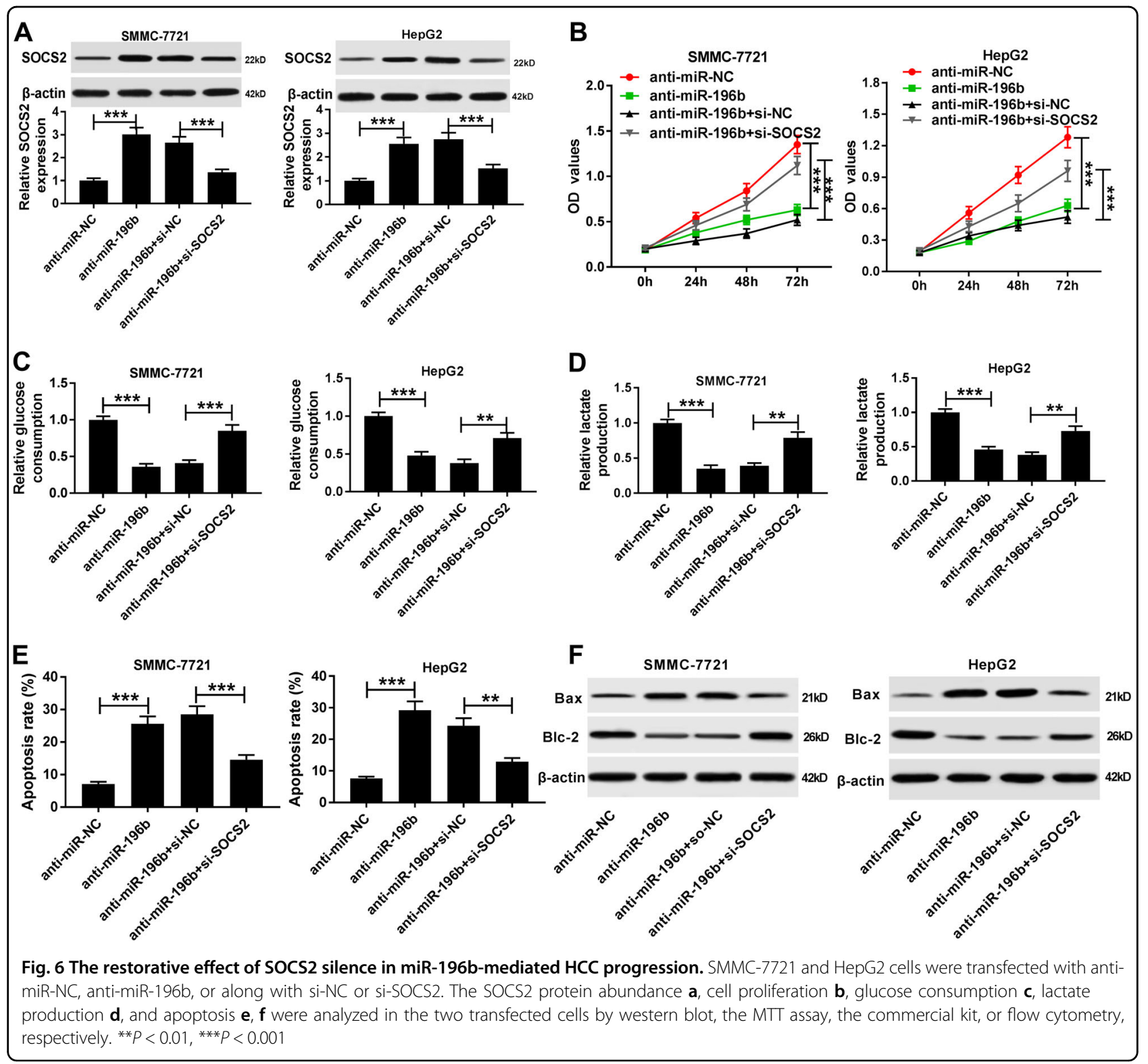

exhibited in Lenti-anti-miR-196a or Lenti-anti-miR196b compared with that in Lenti-anti-miR-NC group, respectively (Fig. 8c). Subsequently, the abundances of p-STAT5, STAT5, p-JAK2, JAK2, and SOCS2 proteins were measured in tumor tissues. Results revealed that inhibition of miR-196a or miR-196b promoted SOCS2 protein level but blocked phosphorylation of JAK2 and STAT5 (Fig. 8d). In addition, tissues showed more apoptotic cells and less cell proliferation in Lenti-antimiR-196a or Lenti-anti-miR-196b compared with that in Lenti-anti-miR-NC group, respectively (Fig. 8e). Besides, knockdown of miR-196a and miR-196b led to obvious decrease of $\mathrm{Bcl}-2$ protein level and increase of Bax protein abundance in xenograft tumor tissues (Fig. S6A and S6B).

\section{Discussion}

miRNAs have been reported to play pivotal roles in regulating development, diagnosis, and antitumor treatments of $\mathrm{HCC}^{21}$. In this study, we found that miR-196a and miR-196b expressions were enhanced in HCC tissues and cells, which is also in agreement with previous efforts $^{10,11}$. This indicated that miR-196a and miR-196b might serve as oncogenes to promote HCC progression. However, the exact roles of miR-196a and miR-196b in HCC remain largely unknown. Here we explored the effect of miR-196a or miR-196b on HCC cell progression and first revealed the mechanism possibly associated with the SOCS2/JAK2/STAT5 signaling pathway.

The former finding has suggested that miR-196a knockdown inhibited cell proliferation, colony formation, 

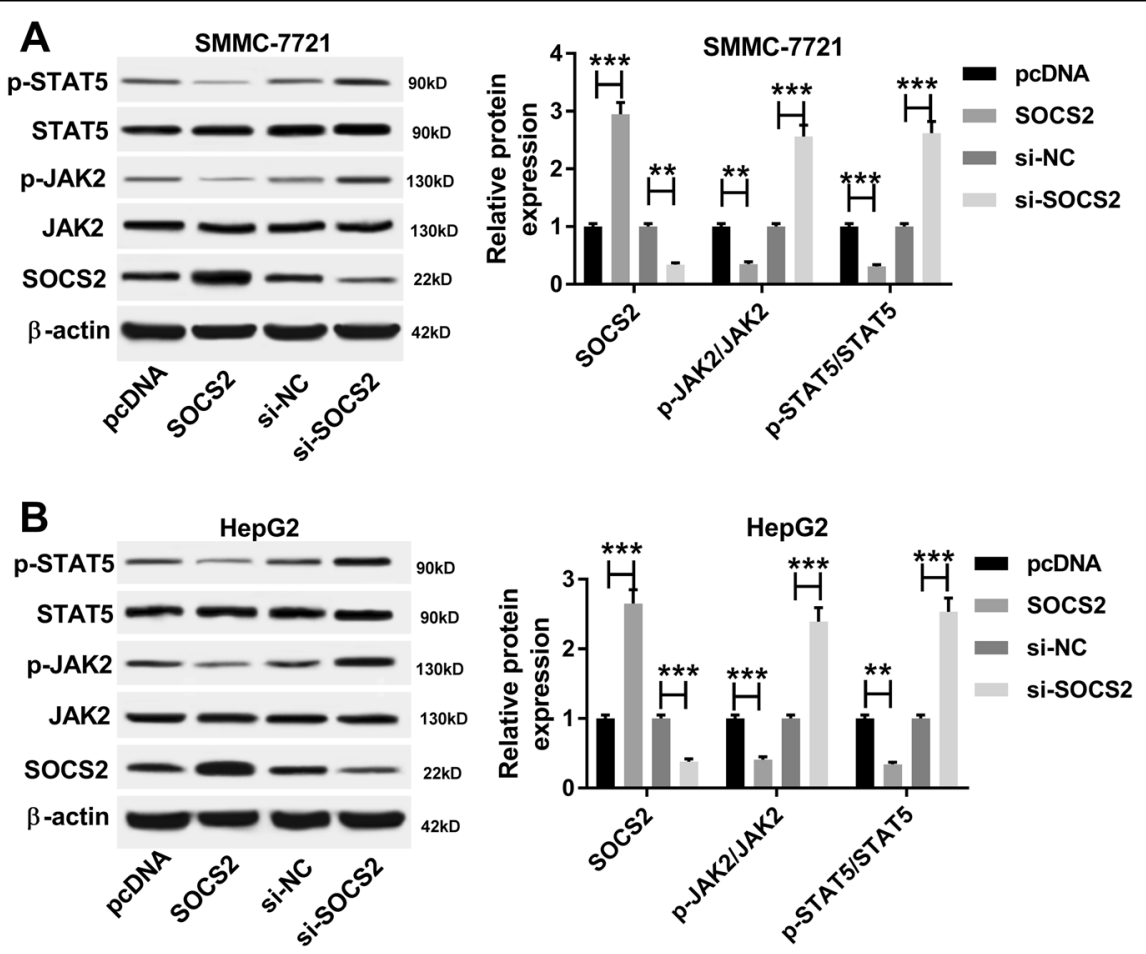

Fig. 7 The effect of SOCS2 on the JAK/STAT pathway in HCC cells. $\mathbf{a}$, $\mathbf{b}$ The expressions of p-STAT5, STAT5, p-JAJ2, JAK2, and SOCS2 protein were measured in SMMC-7721 and HepG2 cells transfected with SOCS2, pCDNA, si-SOCS2, or si-NC by western blot. ${ }^{* *} P<0.01,{ }^{* * *} P<0.001$

migration, and invasion but induced cell apoptosis by targeting forkhead transcription factor O1 (FOXO1) in $\mathrm{HCC}^{22}$. Moreover, miR-196a was reported to contribute to cell proliferation through regulating cell cycle via targeting FOXO1 in $\mathrm{HCC}^{23}$. As for miR-196b, it was shown to predict poor prognosis and increase cell migration as well as invasion in $\mathrm{HCC}$ by regulating FOXP2 ${ }^{24}$. Consistently with these reports, we also found that miR-196a and miR196b knockdown could induce proliferation and apoptosis inhibition and indicate poor outcome of patients. Cell cycle process and colony formation are necessary for normal proliferation, while in this study the two miRNAs knockdown arrested cell cycle process and colony formation. Moreover, elevated glycolysis is an important progress in HCC development, which could support metabolic needs for rapid proliferation of cancer cells ${ }^{25}$. The intrinsic apoptosis pathway is addressed by proapoptotic protein Bax and antiapoptotic protein $\mathrm{Bcl}-2$. The disturbance of $\mathrm{Bcl}-2 / \mathrm{Bax}$ balance contributes to apoptosis production. Our results revealed that knockdown of miR196a and miR-196b inhibited glycolysis and Bcl-2 expression but promoted Bax level. These all findings suggested the poor outcomes of miR-196a and miR-196b in prognosis and progression of $\mathrm{HCC}$.

Functional miRNAs are known to regulate targets expressions by binding their $3^{\prime}$-UTR sequences ${ }^{26}$. This research explored multiple targets of miR-196a and miR- 196b and previous studies have validated the interaction between them and FOXO1, FOXP2, or lysosomeassociated protein transmembrane- $4 \beta$ (LAPTM4B) in $\mathrm{HCC}^{22-24,27}$. SOCS proteins mediate inflammation and apoptosis via regulating immune homeostasis in human cancers. Although Zhao et al. have revealed SOCS2 as a target of miR-196b in human laryngeal squamous cell carcinoma $^{28}$, here we focused on the interaction between miR-196a or miR-196b and SOCS2, which displayed higher miRNA-bound ability than SOCS1. Increasing evidences demonstrated that SOCS2 might function as a tumor suppressor in HCC by regulating tumor progres$\operatorname{sion}^{29-32}$. By luciferase activity and RIP assays we identified SOCS2 as a functional target of miR-196a and miR196b in HCC cells. And recuse experiment by further SOCS2 silence uncovered that miR-196a and miR-196b regulated HCC progression by targeting SOCS2.

Active JAKs recruit STAT proteins to mediate gene transcription, participating in cell proliferation, apoptosis and glucose metabolism. SOCS2 along with other members of SOCS family are regarded as importantly negative regulator of the JAK/STAT pathway ${ }^{33}$. Although STAT3 signaling might be more important than STAT5 in HCC progression and miR-196b could activate STAT3 by targeting SOCS2 in macrophages ${ }^{34,35}$, previous work showed that importance of JAK2/STAT5 signaling in liver metabolism, liver diseases, and $\mathrm{HCC}^{36}$. Hence, we 

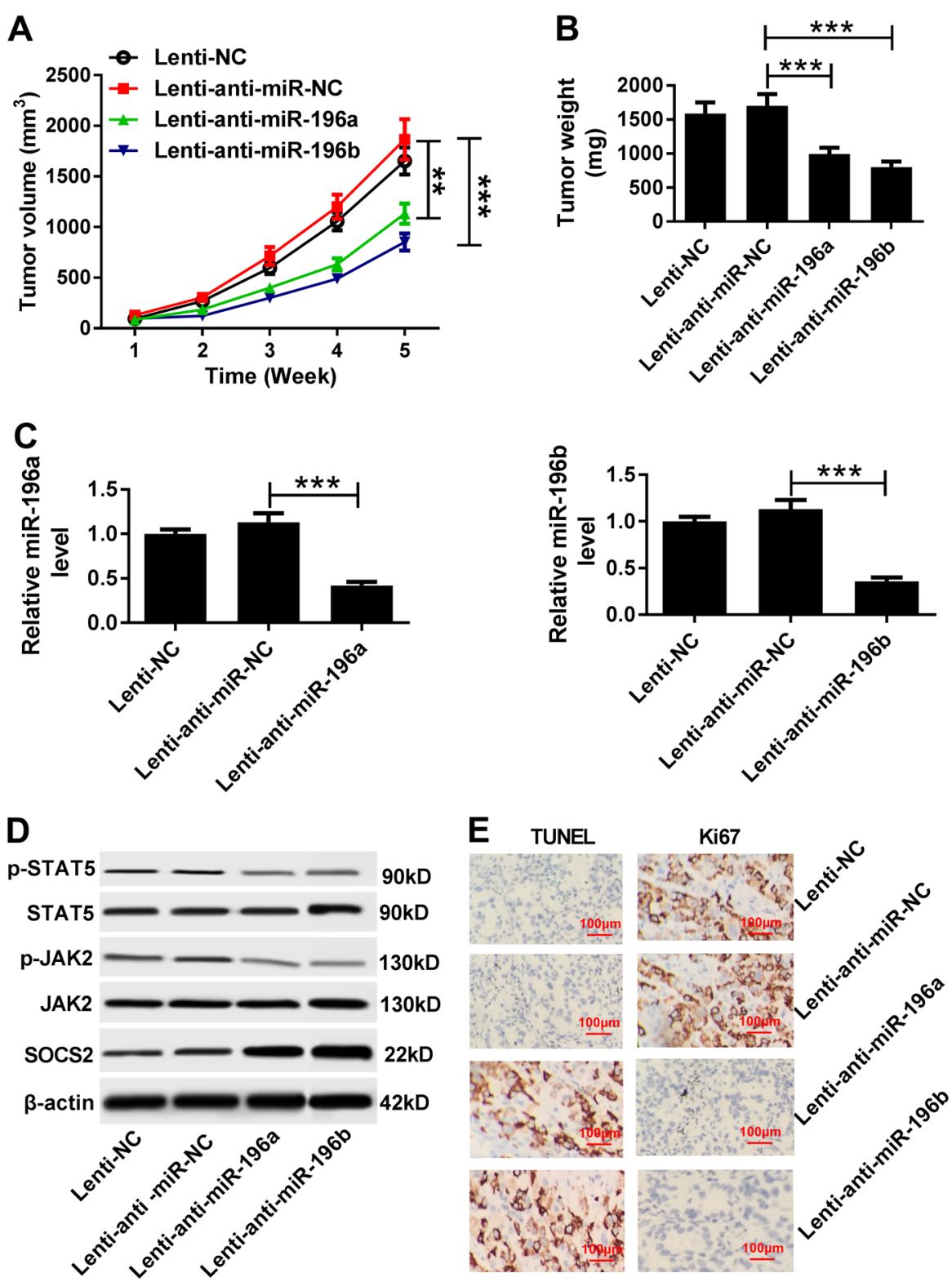

Fig. 8 The effect of miR-196a or miR-196b abrogation on xenograft tumor growth. HepG2 cells were transfected with Lenti-NC, Lenti-anti-miRNC, Lenti-anti-miR-196a, or Lenti-anti-miR-196b. Then stably transfected cells were subcutaneously injected into the nude mice. a Tumor volume was measured every week for 5 weeks. b Tumor weight was detected at ending point. c The expression of miR-196a or miR-196b was examined in tumor tissues by qRT-PCR. $\mathbf{d}$ The protein expressions in the JAK2/STAT5 pathway were analyzed in each group by western blot. e Cell apoptosis and proliferation were investigated in tumor tissues by TUNEL or Ki67 immunohistochemistry. ${ }^{* *} P<0.01,{ }^{* * *} P<0.001$

assumed JAK/STAT signaling was implicated in miR196a- or miR-196b-mediated progression of HCC. This study uncovered that SOCS2 negatively regulated activation of the JAK/STAT pathway in HCC cells and miR196a or miR-196b inhibition decreased HCC progression by regulating the SOCS2/JAK/STAT pathway revealed by a xenograft model.

In conclusion, high expressions of miR-196a and miR196b were shown in HCC and predicted poor outcome of patients. Knockdown of miR-196a or miR-196b inhibited HCC progression, possibly by the SOCS2/JAK2/STAT5 pathway. This study indicates that miR-196a and miR196b might serve as promising therapeutic therapy for HCC treatment.

\section{Acknowledgments}

This work was funded by the Henan Basic and Frontier Technology Research Program (Grant no. 152300410007). 


\section{Author details}

${ }^{1}$ Medical Diagnostic Centre, Luoyang Central Hospital Affiliated to Zhengzhou University, Luoyang, China. ${ }^{2}$ Department of Anesthesiology, Luoyang Central Hospital Affiliated to Zhengzhou University, Luoyang, China. ${ }^{3}$ Department of Orthopedics, Luoyang Central Hospital Affiliated to Zhengzhou University, Luoyang, China

\section{Data availability}

The datasets from current study are available from the corresponding author on reasonable request.

\section{Ethical approval and consent to participate}

Written informed consent was obtained from all participants and the work was accepted by the Research Ethics Committee of Luoyang Central Hospital Affiliated to Zhengzhou University. Every experiment was conducted under the approval of the Animal Research Committee of Luoyang Central Hospital Affiliated to Zhengzhou University.

\section{Conflict of interest}

The authors declare that they have no conflict of interest.

\section{Publisher's note}

Springer Nature remains neutral with regard to jurisdictional claims in published maps and institutional affiliations.

Supplementary Information accompanies this paper at (https://doi.org/ 10.1038/s41419-019-1530-4)

Received: 5 December 2018 Revised: 8 March 2019 Accepted: 12 March 2019

Published online: 15 April 2019

\section{References}

1. Forner, A., Reig, M. \& Bruix, J. Hepatocellular carcinoma. Lancet 391, 1301-1314 (2018).

2. Bruix, J., Reig, M. \& Sherman, M. Evidence-based diagnosis, staging, and treatment of patients with hepatocellular carcinoma. Gastroenterology $\mathbf{1 5 0}$ 835-853 (2016).

3. $\mathrm{Xu}, \mathrm{X}$. et al. The role of microRNAs in hepatocellular carcinoma. J. Cancer $\mathbf{9}$ 3557-3569 (2018)

4. Chen, C., Zhang, Y., Zhang, L., Weakley, S. \& Yao, Q. MicroRNA-196: critical roles and clinical applications in development and cancer. J. Cell. Mol. Med. 15, 14-23 (2011).

5. Guan, Y. et al. MiRNA-196 is upregulated in glioblastoma but not in anaplastic astrocytoma and has prognostic significance. Clin. Cancer Res. 16, 4289-4297 (2010).

6. Li, Y. et al. Ratio of miR-196s to HOXC8 messenger RNA correlates with breast cancer cell migration and metastasis. Cancer Res. 70, 7894-7904 (2010).

7. Lu, Y. C. et al. OncomiR-196 promotes an invasive phenotype in oral cancer through the NME4-JNK-TIMP1-MMP signaling pathway. Mol. Cancer 13, 218 (2014).

8. Lu, Y. C. et al. miR-196, an emerging cancer biomarker for digestive tract cancers. J. Cancer 7, 650-655 (2016).

9. Tsai, M. M. et al. MicroRNA-196a/-196b promote cell metastasis via negative regulation of radixin in human gastric cancer. Cancer Lett. 351, 222-231 (2004).

10. Li, J. et al. Analysis of microRNA expression profiles in human hepatitis B virusrelated hepatocellular carcinoma. Clin. Lab. 59, 1009-1015 (2013).

11. Shen, J. et al. Genome-wide aberrant DNA methylation of microRNA host genes in hepatocellular carcinoma. Epigenetics 7, 1230-1237 (2012).

12. Vasuri, F. et al. Role of microRNAs in the main molecular pathways of hepatocellular carcinoma. World J. Gastroenterol. 24, 2647-2660 (2018).

13. Gao, B., Wang, H., Lafdil, F. \& Feng, D. STAT proteins: key regulators of anti-viral responses, inflammation, and tumorigenesis in the liver. J. Hepatol. $\mathbf{5 7}$ 430-441 (2012)
14. Xu, G. et al. MiR-221 accentuates IFN's anti-HCV effect by downregulating SOCS1 and SOCS3. Virology 462, 343-350 (2014).

15. Wonganan, O. et al. 6-Hydroxy-3-O-methyl-kaempferol 6-O-glucopyranoside potentiates the anti-proliferative effect of interferon $\alpha / \beta$ by promoting activation of the JAKSSTAT signaling by inhibiting SOCS3 in hepatocellular carcinoma cells. Toxicol. Appl. Pharmacol. 336, 31-39 (2017).

16. $Y u, Y$. et al. Hepatitis B virus e antigen activates the suppressor of cytokine signaling 2 to repress interferon action. Sci. Rep. 7, 1729 (2017).

17. Rico-Bautista, E., Flores-Morales, A. \& Fernández-Pérez, L. Suppressor of cytokine signaling (SOCS) 2, a protein with multiple functions. Cytokine Growth Factor Rev. 17, 431-439 (2006).

18. Johnson, P. J. et al. Assessment of liver function in patients with hepatocellular carcinoma: a new evidence-based approach-the ALBI grade. J. Clin. Oncol. 33 550-558 (2015)

19. Livak, K. J. \& Schmittgen, T. D. Analysis of relative gene expression data using real-time quantitative PCR and the 2(-Delta Delta C(T)) Method. Methods 25, 402-408 (2001)

20. Seyhoun, I. et al. Combination therapy of sorafenib with mesenchymal stem cells as a novel cancer treatment regimen in xenograft models of hepatocellular carcinoma. J. Cell. Physiol. 234, 9495-9503 (2019).

21. Pan, J. H. et al. Role of exosomes and exosomal microRNAs in hepatocellular carcinoma: potential in diagnosis and antitumour treatments (Review). Int. J. Mol. Med. 41, 1809-1816 (2018).

22. Yang, L, Peng, F, Oin, J, Zhou, $H$. \& Wang, B. Downregulation of microRNA$196 a$ inhibits human liver cancer cell proliferation and invasion by targeting FOXO1. Oncol. Rep. 38, 2148-2154 (2017).

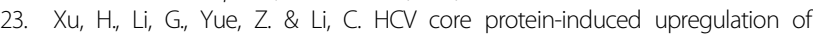
microRNA-196a promotes aberrant proliferation in hepatocellular carcinoma by targeting FOXO1. Mol. Med. Rep. 13, 5223-5229 (2016).

24. Yu, Z., Lin, X., Tian, M. \& Chang, W. microRNA-196b promotes cell migration and invasion by targeting FOXP2 in hepatocellular carcinoma. Oncol. Rep. 39 731-738 (2018).

25. $\mathrm{Xu}, \mathrm{Q}$. et al. Oviductus ranae protein hydrolysate $(\mathrm{ORPH})$ inhibits the growth metastasis and glycolysis of HCC by targeting miR-491-5p/PKM2 axis. Biomed. Pharmacother. 107, 1692-1704 (2018).

26. Bartel, D. P. MicroRNAs: target recognition and regulatory functions. Cell 136, 215-233 (2009).

27. Xie, C. R. et al. Long noncoding RNA HCAL facilitates the growth and metastasis of hepatocellular carcinoma by acting as a ceRNA of LAPTM4B. Mol. Ther Nucleic Acids 9, 440-451 (2017).

28. Zhao, X., Zhang, W. \& Ji, W. miR-196b is a prognostic factor of human laryngeal squamous cell carcinoma and promotes tumor progression by targeting SOCS2. Biochem. Biophys. Res. Commun. 501, 584-592 (2018)

29. Li, B. et al. Development and validation of a three-gene prognostic signature for patients with hepatocellular carcinoma. Sci. Rep. 7, 5517 (2017).

30. Chen, M. et al. RNA N6-methyladenosine methyltransferase-like 3 promotes liver cancer progression through YTHDF2-dependent posttranscriptional silencing of SOCS2. Hepatology 67, 2254-2270 (2018).

31. Long, J. et al. Maintenance of stemness by miR-589-5p in hepatocellular carcinoma cells promotes chemoresistance via STAT3 signaling. Cancer Lett. 423, 113-126 (2018)

32. Qiu, X. et al. Reduced expression of SOCS2 and SOCS6 in hepatocellular carcinoma correlates with aggressive tumor progression and poor prognosis. Mol. Cell. Biochem. 378, 99-106 (2013).

33. Almeida-Oliveira, A. R et al. Effects of aerobic exercise on molecular aspects of asthma: involvement of SOCS-JAK-STAT. Exerc. Immunol. Rev. 25, 50-62 (2019).

34. Rajendran, P. et al. $\mathrm{Y}$-Tocotrienol is a novel inhibitor of constitutive and inducible STAT3 signalling pathway in human hepatocellular carcinoma: potential role as an antiproliferative, pro-apoptotic and chemosensitizing agent. Br. J. Pharmacol. 163, 283-298 (2011).

35. Yuan, $Y$. et al. Upregulation of miR-196b-5p attenuates BCG uptake via targeting SOCS3 and activating STAT3 in macrophages from patients with longterm cigarette smoking-related active pulmonary tuberculosis. J. Transl. Med. 16, 284 (2018).

36. Kaltenecker D., et al. Hepatic growth hormone - JAK2 - STAT5 signalling: metabolic function, non-alcoholic fatty liver disease and hepatocellular carcinoma progression. Cytokine https://doi.org/10.1016/j.cyto.2018.10.010 (2018) 